\title{
Isomorfismo cardiaco: Una perspectiva multidisciplinaria
}

\author{
Atrial isomerism: A multidisciplinary perspective \\ Diego B. Ortega-Zhindón ${ }^{1}$, Iris P. Flores-Sarria1, María A. Minakata-Quiróga², \\ Stephanie T. Angulo-Cruzado 3 , Luis A. Romero-Montalvo y Jorge L. Cervantes-Salazar * \\ ${ }^{1}$ Departamento de Cirugía Cardiaca Pediátrica y Cardiopatías Congénitas; ${ }^{2}$ Departamento de Anestesia Cardiovascular; ${ }^{3}$ Departamento de \\ Cardiopatías Congénitas en el Adulto; ${ }^{4}$ Departamento de Cardiología Intervencionista en Cardiopatías Congénitas. Instituto Nacional de Cardiología \\ Ignacio Chávez, Ciudad de México, México
}

\begin{abstract}
Resumen
El isomorfismo cardiaco describe hallazgos anatómicos complejos con defectos en la determinación de la lateralización. Es una situación poco frecuente, con prevalencia de 1 en cada 10,000 a 20,000 nacidos vivos, con incidencia hasta del 4\% de todas las malformaciones cardiacas. El diagnóstico puede realizarse en la etapa neonatal; sin embargo, el cuadro clínico es inespecífico. De acuerdo con el espectro de malformaciones se pueden necesitar medios diagnósticos complejos e invasivos. El tratamiento es variado y puede ir desde la cirugía paliativa en vista de una fisiología univentricular hasta una cirugía de corrección total para una reparación biventricular.
\end{abstract}

Palabras clave: Síndrome de heterotaxia. Isomerismo auricular. Cardiopatía congénita

\begin{abstract}
Atrial isomerism describes complex anatomical findings with defects in the determination of lateralization; being a rare situation, with a prevalence of 1 in every 10.000 to 20.000 live births, with an incidence of up to $4 \%$ of all cardiac malformations. The diagnosis can be made in the neonatal age; however, clinical presentation is nonspecific. Depending on the spectrum of malformations, complex and invasive diagnostic tools may be required. Treatment is varied and can range from palliative surgery in view of univentricular physiology to total correction surgery for biventricular repair.
\end{abstract}

Key words: Heterotaxy syndrome. Atrial isomerism. Congenital heart defect.

\section{Introducción}

Isomorfismo cardiaco es un término producto de la evolución del compendio morfológico a lo largo del tiempo de su estudio y surge al complementarse dos términos. Heterotaxia, derivado del griego hetero, que significa «diferente» y taxi, que significa «disposición».
Denota la disposición anormal de los órganos toracoabdominales internos a través del eje izquierdo-derecho del cuerpo sin que existan alteraciones cardiacas, por lo que llevó a la denominación de los síndromes de asplenia o poliesplenia ${ }^{1,2}$. Por otro lado, isomerismo, palabra derivada del griego iso, que significa «igual» $y$

\section{Correspondencia:}

*Jorge L. Cervantes-Salazar

E-mail: jorgeluis.cervantes @gmail.com
Fecha de recepción: 19-12-2020

Fecha de aceptación: 17-03-2021

DOI: 10.24875/ACM.20000567
Disponible en internet: 06-09-2021 Arch Cardiol Mex. 2021;91(4):470-479 www.archivoscardiologia.com

1405-9940 / @ 2021 Instituto Nacional de Cardiología Ignacio Chávez. Publicado por Permanyer. Este es un artículo open access bajo la licencia CC BY-NC-ND (http://creativecommons.org/licenses/by-nc-nd/4.0/). 
meros, que significa «parte», se adoptó considerando que en pacientes con heterotaxia se pierde la asimetría normal de los órganos torácicos y abdominales, dando como resultado una simetría de las estructuras morfológicas que normalmente se desarrollan en cada lado ${ }^{1,2}$. Por lo tanto, estos términos son no excluyentes; sin embargo, la connotación dada por las ciencias fisicoquímicas al término «isomérico» se relaciona con la ubicación espacial de una estructura de este campo, por lo que el término que considerar como adecuado en relación con el componente morfológico y al estar relacionado con la simetría auricular es el de isomorfismo cardiaco.

\section{Epidemiología}

Las malformaciones cardiacas en los recién nacidos vivos de manera global alcanza el $0.8 \%$, como probable consecuencia de la herencia familiar y su polimorfismo; sin embargo, hasta el momento no se ha definido de forma exacta su origen, atribuyéndose a una causa multifactorial ${ }^{3}$. Es así como la prevalencia puede variar de región a región con reportes desde 2.1 hasta 12.3 por 1,000 nacidos vivos ${ }^{4}$ y con una incidencia de 6 a 8 por cada 1,000 nacidos vivos ${ }^{5}$. Dentro de las múltiples cardiopatías congénitas resalta el isomorfismo cardiaco como una entidad compleja que se acompaña de un espectro de malformaciones cardiacas y extracardiacas, mostrando ser una entidad poco frecuente, con una prevalencia de 1 en cada 10,000 a 20,000 nacidos vivos y con una incidencia hasta del $4 \%$ de todas las malformaciones cardiacas ${ }^{6}$.

\section{Consideraciones embriológicas y anatómicas}

Al final de la tercera semana, durante el periodo de diferenciación de las células cardiacas y a partir del nódulo de Hensen se lleva a cabo el desarrollo y lateralización de las estructuras derechas e izquierdas ${ }^{3,6}$, no obstante, en los pacientes con isomorfismo existen patrones de expresión génica, principalmente involucrando a los genes Nodal y Pitx2, además de otros como NKX2-5, CRELD1, LEFTY2, ZIC3 y CRIPTC; que se asocian a la codificación de los componentes de la vía del factor de crecimiento transformante beta (TGF- $\beta$, transforming growth factor beta), que al verse alterada determina una de las dos entidades: dextromorfismo o levomorfismo ${ }^{3}$.

En el dextromorfismo, las estructuras se conforman en lados opuestos del eje izquierdo-derecho del cuerpo como imágenes en espejo, simétricas entre sí con morfología de estructuras del lado derecho. Así, las dos aurículas adquieren una morfología derecha, con ausencia de estructuras laterales como el seno coronario, por ejemplo. A esto se suman otras malformaciones extracardiacas como pulmones trilobulados bilateralmente y de estos cada uno con un bronquio corto, la ausencia de bazo, malrotación intestinal, entre otras $^{1,3,6,7}$.

Por otro lado, en el levomorfismo, las estructuras emparejadas en lados opuestos del eje izquierdo-derecho del cuerpo son imágenes en espejo, simétricas entre sí que adoptan la morfología de las estructuras normales del lado izquierdo, por lo que las dos aurículas y apéndices adquieren la morfología izquierda. Otras alteraciones cardiovasculares que se pueden observar es la ausencia o interrupción de la vena cava inferior hasta en el $80 \%$ de los casos y, aunque la ausencia de seno coronario es más frecuente en el dextromorfismo, hasta un $50 \%$ de los casos pueden tenerlo. Así mismo, aunque con menor frecuencia que en el dextromorfismo, el drenaje pulmonar venoso anómalo y el canal auriculoventricular completo pueden estar presentes y en estos casos es más probable encontrar corazones con fisiología biventricular (Tabla 1). Las malformaciones extracardiacas que puede presentar son pulmones bilobulados bilateralmente cada uno con un bronquio largo y poliesplenia, entre otras $^{1,3,6-8}$.

\section{Hallazgos clínicos}

La presentación clínica puede variar de un sujeto a otro, desde formas casi imperceptibles como la interrupción de la vena cava inferior hasta formas graves y potencialmente mortales como la obstrucción del drenaje venoso pulmonar ${ }^{1}$, resultando esta en una situación critica ${ }^{9,10}$ y un factor de riesgo importante para mortalidad (hazard ratio [HR]: 4.40; $p=0.010)^{9}$. La evaluación inicial, principalmente en la etapa neonatal, es importante en el contexto de una decisión y asesoramiento fetal, así como para la atención posnatal temprana $^{10}$, que determine la derivación a las diferentes especialidades involucradas. No obstante, el reconocimiento de ciertos signos y síntomas nos llevará a la sospecha de la entidad clínica, por ejemplo si se evidencia cianosis, al ser la forma de presentación más común, puede acercarnos hacia un probable dextromorfismo que en ocasiones resulta de un drenaje venoso pulmonar anómalo obstruido ${ }^{1,2,6}$, pero también la asociación de otros problemas como disnea y soplo 
Tabla 1. Características del isomorfismo cardiaco

\begin{tabular}{|c|c|c|}
\hline Características & Levomorfismo & Dextromorfismo \\
\hline $\begin{array}{l}\text { Apéndices } \\
\text { auriculares }\end{array}$ & $\begin{array}{l}\text { AD: apéndice auricular izquierdo } \\
\text { Al: apéndice auricular izquierdo }\end{array}$ & $\begin{array}{l}\text { AD: apéndice auricular derecho } \\
\text { Al: apéndice auricular derecho }\end{array}$ \\
\hline $\begin{array}{l}\text { Ápex cardiaco } \\
\text { (variable) }\end{array}$ & Levocardia & Levocardia \\
\hline Aurículas & $\begin{array}{l}\text { Aurícula común, defectos septales auriculares, mal posición del } \\
\text { septo interauricular }\end{array}$ & Ausencia de seno coronario \\
\hline $\begin{array}{l}\text { Conexión } \\
\text { auriculoventricular }\end{array}$ & $\begin{array}{l}\text { Biventricular, } 2 \text { válvulas AV separadas o puede encontrarse } \\
\text { válvula AV única, canal AV común, vías de conducción accesorias }\end{array}$ & $\begin{array}{l}\text { Univentricular, válvula AV única, canal AV } \\
\text { común }\end{array}$ \\
\hline $\begin{array}{l}\text { Concordancia } \\
\text { auriculoventricular }\end{array}$ & Generalmente concordante & Posible discordancia \\
\hline Ventrículos & Generalmente normal & Defectos septales ventriculares \\
\hline $\begin{array}{l}\text { Conexión } \\
\text { ventriculoarterial }\end{array}$ & $\begin{array}{l}\text { Generalmente normal. Posibles malformaciones: transposición de } \\
\text { grandes arterias, doble salida del ventrículo derecho, estenosis } \\
\text { subaórtica, estenosis subpulmonar }\end{array}$ & $\begin{array}{l}\text { Estenosis subaórtica, estenosis } \\
\text { subpulmonar, doble salida del ventrículo } \\
\text { derecho }\end{array}$ \\
\hline $\begin{array}{l}\text { Concordancia } \\
\text { ventriculoarterial }\end{array}$ & Generalmente concordante & Posible discordancia \\
\hline Arteriales & $\begin{array}{l}\text { Arco aórtico izquierdo, interrupción del arco aórtico, relación } \\
\text { anómala entre aorta abdominal y vena cava inferior }\end{array}$ & Alteraciones del arco aórtico \\
\hline Venosas & $\begin{array}{l}\text { Anomalías venosas sistémicas: interrupción o ausencia de vena } \\
\text { cava inferior, continuación de vena cava inferior con sistema } \\
\text { ácigos, vena cava superior bilateral }\end{array}$ & $\begin{array}{l}\text { Anomalías venosas pulmonares: drenaje } \\
\text { venoso pulmonar anómalo parcial o total }\end{array}$ \\
\hline $\begin{array}{l}\text { Anomalías } \\
\text { bronquiales }\end{array}$ & Isomorfismo bronquial izquierdo & Isomorfismo bronquial derecho \\
\hline $\begin{array}{l}\text { Anomalías } \\
\text { pulmonares }\end{array}$ & 2 pulmones bilobulados & 2 pulmones trilobulados \\
\hline $\begin{array}{l}\text { Anomalías } \\
\text { extratorácicas }\end{array}$ & $\begin{array}{l}\text { Poliesplenia, malrotación intestinal, hígado invertido, atresia biliar } \\
\text { extrahepática }\end{array}$ & $\begin{array}{l}\text { Asplenia, malrotación intestinal, estómago } \\
\text { hacia la derecha, hígado simétrico }\end{array}$ \\
\hline
\end{tabular}

$\mathrm{AD}$ : aurícula derecha; Al: aurícula izquierda; $\mathrm{AV}$ : auriculoventricular.

cardiaco nos podría hacer sospechar en una estenosis pulmonar asociada ${ }^{1}$. En relación con el levomorfismo, los síntomas generalmente se presentan en una edad más tardía, debido a una menor gravedad de las lesiones asociadas, como obstrucciones leves a nivel de la arteria pulmonar; pero pueden dar manifestaciones de cronicidad como taquipnea en forma secundaria a una insuficiencia cardiaca ya instaurada, o por otro lado encontrarse asintomáticos e iniciar con problemas asociados a una malformación extracardiaca ${ }^{1,2,6,8}$ (Tabla 1). Aunque se ha considerado a la ausencia de bazo o presencia de múltiples bazos accesorios como parte complementaria para el diagnóstico de esta patología, se ha determinado en los últimos reportes que la variedad de presentación en cada grupo es diversa, donde se puede encontrar asplenia entre el 68 y el $79 \%$ de los pacientes con dextromorfismo y para el levomorfismo la poliesplenia puede estar entre el 84 y el $88 \% 9$; por esta razón, el predominio de procesos infecciosos se da en pacientes con dextromorfismo, principalmente por bacterias encapsuladas, considerado como un factor predictor de mortalidad (HR: $1.67 ; p=0.044)^{10}$.

\section{Métodos diagnósticos}

Con el advenimiento de varios métodos no invasivos de imagen se puede hacer un diagnóstico prenatal desde la semana 22 a 24 de gestación $6,11,12$. El primer abordaje frecuentemente se hace con el apoyo de estudios como radiografía de tórax, electrocardiograma y ecocardiograma; posteriormente, de acuerdo con la variabilidad de malformaciones que tenga el paciente, se complementa con abordajes diagnósticos más complejos e invasivos.

Con seguridad la radiografía de tórax es el primer acercamiento con el que podemos determinar un situs 
solitus o diferenciarlo de otras variedades como situs inversus o situs ambiguous; por otro lado, la morfología de los bronquios y de las estructuras abdominales nos permite dar una primera aproximación por este método 6 .

El electrocardiograma generalmente presenta trazos anormales, debido a los defectos anatómicos en el nodo sinusal y nodo auriculoventricular, además de una despolarización anómala de los ventrículos por defectos en el tejido de conducción ${ }^{1,6}$. En el dextromorfismo la actividad de los nodos sinusales bilaterales se puede presentar como morfologías de ondas $\mathrm{P}$ múltiples, de origen derecho e izquierdo o un ritmo auricular ectópico, así como mayor propensión a fibrilación auricular y flutter auricular ${ }^{1,6,7}$. En el levomorfismo debido a la hipoplasia del nodo sinusal puede no existir un verdadero ritmo sinusal ${ }^{1,7,8}$, teniendo un eje de onda $P$ dirigido hacia arriba y a la izquierda con un ritmo auricular ectópico, y algunas veces en pacientes de mayor edad puede existir una disminución de la frecuencia cardiaca o bloqueos auriculoventriculares, situación rara en el dextromorfismo $0^{1,8}$.

El ecocardiograma constituye el principal método diagnóstico inicial ${ }^{13}$, tanto en la etapa prenatal como en la posnatal, debido a la precisión que puede tener en relación con las imágenes anatómicas. Recientemente se identificó en la etapa fetal persistencia de la vena umbilical derecha en el $42 \%$ de casos con isomorfismo, predominando en el dextromorfismo con un $73 \%{ }^{14}$. En la etapa posnatal el isomorfismo derecho o izquierdo se diagnostica por la posición anormal de la vena cava inferior y la aorta abdominal, además de múltiples asociaciones con otros defectos cardiacos ${ }^{1,6,8}$ (Tabla 1).

Las imágenes por resonancia magnética cardiaca (RMC) y tomografía computarizada (TC) son seguras y efectivas, complementando las limitaciones del ecocardiograma transtorácico para valoración de estructuras vasculares extracardiacas, valoración anatómica y relación de los bronquios con las arterias pulmonares e imagen simultánea de ambos apéndices atriales y delineación de la anatomía abdominal (incluyendo al bazo).

La RMC brinda información funcional y hemodinámica útil, permitiendo cuantificación de volúmenes y función ventricular, así como datos anatómicos que permitan una planeación quirúrgica cuidadosa ${ }^{15}$. Por otro lado, el uso de TC será adecuado para evaluación de anatomía compleja en neonatos o lactantes, anatomía extracardiaca (parénquima pulmonar, vía aérea, etc.), valoración de anatomía de arterias coronarias y en casos de contraindicación para $\mathrm{RMC}^{16}$.

Es importante aplicar el llamado «método morfológico", establecido por van Praagh ${ }^{17}$, con el cual se describe el componente más constante, siendo la mejor forma para definir la morfología auricular, idealmente basado en la disposición de los apéndices auriculares de acuerdo con la extensión de los músculos pectíneos. En este sentido, la valoración inicial considerara al situs, donde describe la posición y configuración de las aurículas, el árbol traqueobronquial y las vísceras abdominales en las cavidades toracoabdominales, clasificándolo como $^{18,19}$ : solitus (posición anatómica normal), inversus (imagen en espejo del situs solitus por inversión de las posiciones visceroatriales dentro del tórax y abdomen) y ambiguous (la lateralización de las aurículas y los órganos viscerales no puede ser definida como solitus o inversus).

El situs atrial está determinado por la morfología auricular basada en las características de sus apéndi$\operatorname{ces}^{17,20}$, teniendo una aurícula morfológicamente derecha cuando la orejuela es piramidal, con presencia de la crista terminalis, y de forma importante, la disposición de unos prominentes músculos pectíneos extendiéndose hacia el vestíbulo de la válvula tricuspídea en toda su extensión parietal ${ }^{21}$ (Fig. $1 \mathrm{~A}$ y C) y generalmente localizada en el mismo lado del hígado, resultando en concordancia del situs visceroatrial ${ }^{22}$; mientras que una aurícula morfológicamente izquierda se ubica en una posición más cefálica en el mediastino con respecto al atrio derecho, además de una orejuela delgada y tubular de cuello estrecho (digitiforme) con ausencia de crista terminalis, tiene el vestíbulo de la válvula mitral siempre confluente con el lado liso del componente venoso pulmonar, es decir, que los músculos pectíneos solo están dentro de la orejuela ${ }^{21}$ (Fig. 1 B y D).

El situs broncopulmonar determinado por la morfología bronquial y la relación de las arterias pulmonares con sus bronquios ipsilaterales (Fig. $2 \mathrm{~A}$ y B) ${ }^{19}$, habitualmente son concordantes con el situs atrial ${ }^{23}$, aunque se reportan casos de disarmonía entre situs broncopulmonar y atrial (2\% en dextromorfismo y entre 4 y el $8 \%$ en levomorfismo $)^{21,24}$. Morfológicamente, un bronquio derecho es más corto y horizontal que el izquierdo, con tres lóbulos y la rama pulmonar derecha cruza anterior y ligeramente inferior a este «bronquio epiarterial» (Fig. 2 C y E); mientras que un bronquio morfológicamente izquierdo es más largo que el derecho $(1.5 \mathrm{a} 2 \mathrm{~cm})$ y la arteria pulmonar izquierda cruza sobre el «bronquio hipoarterial» (Fig. $2 \mathrm{D}$ y F) 22 . 

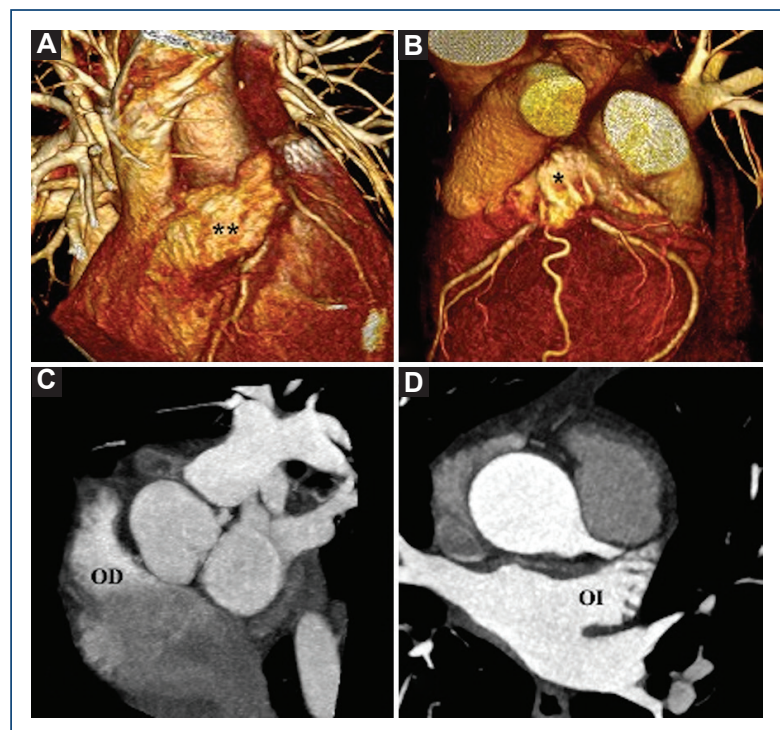

Figura 1. Reconstrucción 3D que muestra una aurícula morfológicamente derecha con $(\mathbf{A})$ orejuela triangular de base ancha y una (B) aurícula morfológicamente izquierda $\left({ }^{*}\right)$ con base angosta y digitiforme. Proyección de máxima intensidad muestra (C) orejuela derecha (OD) y (D) orejuela izquierda (OI) normales.

El situs abdominal normalmente es asimétrico, debido a que en los humanos las vísceras torácicas y abdominales tienen esta disposición y están lateralizadas ${ }^{18}$. En la mayoría de los casos, la disposición atrial en anormalidades del situs es paralela a la disposición de las vísceras abdominales. La posición de la aorta abdominal, vena cava inferior, estómago, bazo e hígado es normal en el situs solitus y reversa en situs inversus (Fig. 3); mientras que en los situs ambiguous (isomorfismo) esa relación es más compleja ${ }^{19}$.

Los pacientes con isomorfismo presentan una duplicación de la morfología auricular y bronquial, con una estructura de imagen en espejo e independiente de la posición cardiaca ${ }^{19,25,26}$. En el dextromorfismo (Fig. 4), las estructuras dentro de la cavidad toracoabdominal tienen características derechas, siendo la principal la morfología derecha de los apéndices auriculares ${ }^{20}$, además de pulmones trilobulados con bronquios epiarteriales bilaterales ${ }^{1,3,6}$, vena cava superior bilateral (46$71 \%$ ), hígado simétrico $(25-76 \%)^{21,24}$, asociado con asplenia en aproximadamente el $42 \%{ }^{1,3,6}$. El bazo es el único órgano mesenquimal que está al lado izquierdo desde su inicio, pues se desarrolla en el lado izquierdo del mesogastrio dorsal ${ }^{27}$, por lo que es frecuente la ausencia de bazo en el dextromorfismo. La conexión anómala total de las venas pulmonares es usual en el

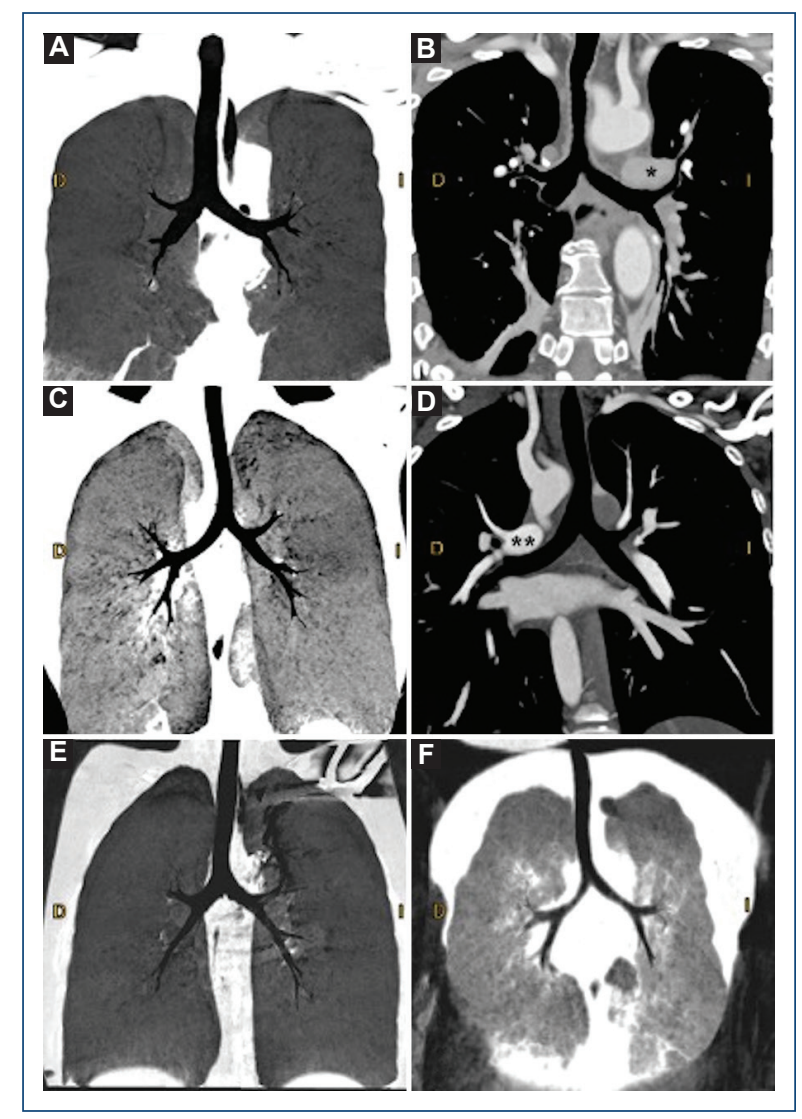

Figura 2. Reconstrucción multiplanar que muestra (A) anatomía bronquial en situs solitus con el bronquio derecho más corto y horizontal que el izquierdo y (B) la rama pulmonar izquierda $\left({ }^{*}\right)$ sobre el bronquio (hipoarterial). Tomografía computarizada con proyección de mínima intensidad que muestra (C) situs inversus atriobronquial con el bronquio a la derecha morfológicamente izquierdo más largo y menos horizontal y el bronquio a la izquierda morfológicamente derecho. Reconstrucción de proyección de máxima intensidad que muestra (D) bronquio a la derecha hipoarterial $\left(^{* *}\right)$. Reconstrucción multiplanar que muestra (A) dextromorfismo bronquial: bronquios cortos y horizontales; levomorfismo bronquial (F): bronquios largos y pulmones bilobulados.

dextromorfismo ${ }^{9}$, esto debido a la presencia de dos aurículas morfológicamente derechas que al determinar la llegada de estas hacia la aurícula de ubicación espacial izquierda pero morfológicamente derecha, resulta por definición en una conexión venosa pulmonar anómala en la totalidad de estos pacientes ${ }^{9,21,24}$. Las conexiones auriculoventriculares son univentriculares 0 biventriculares, en casi la misma proporción (54 vs. $46 \%{ }^{21}$; no obstante, Uemura, et al. (1995) encontraron predominio de válvulas auriculoventriculares comunes en el $92 \%$ de los casos; por otro lado, las conexiones 


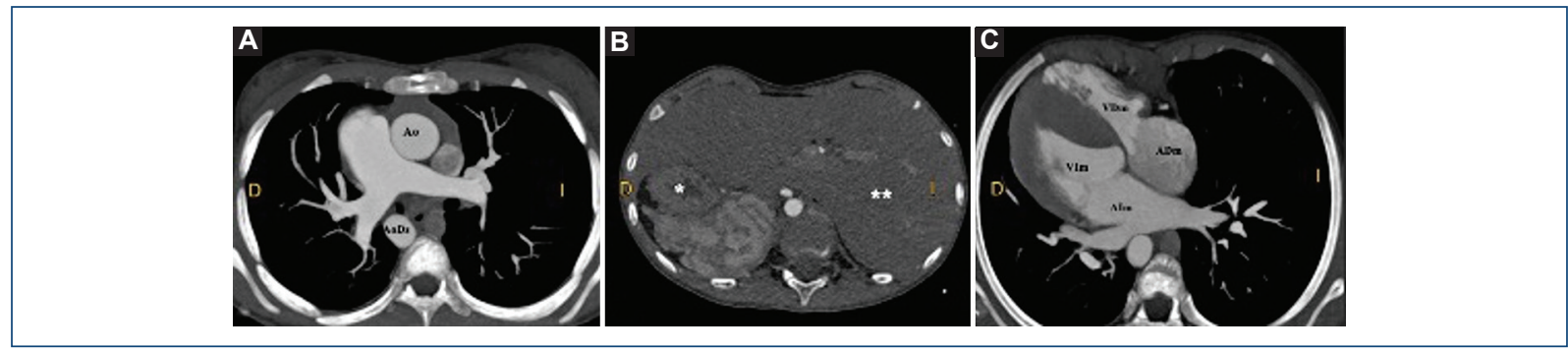

Figura 3. Tomografía computarizada axial con reconstrucción que muestra (A) a nivel de los grandes vasos la aorta descendente hacia la derecha, (B) el hígado $\left({ }^{*}\right)$ a la izquierda y el estómago $\left({ }^{*}\right)$ a la derecha. Vista modificada que muestra (C) cámaras cardiacas en dextrocardia y dextroápex, las cavidades morfológicamente izquierdas situadas a la derecha (imagen en espejo).

ADm: aurícula derecha morfológica; Alm: aurícula izquierda morfológica; Ao: aorta; AoDs: aorta descendente; VDm: ventrículo derecho morfológico; VIm: ventrículo izquierdo morfológico.

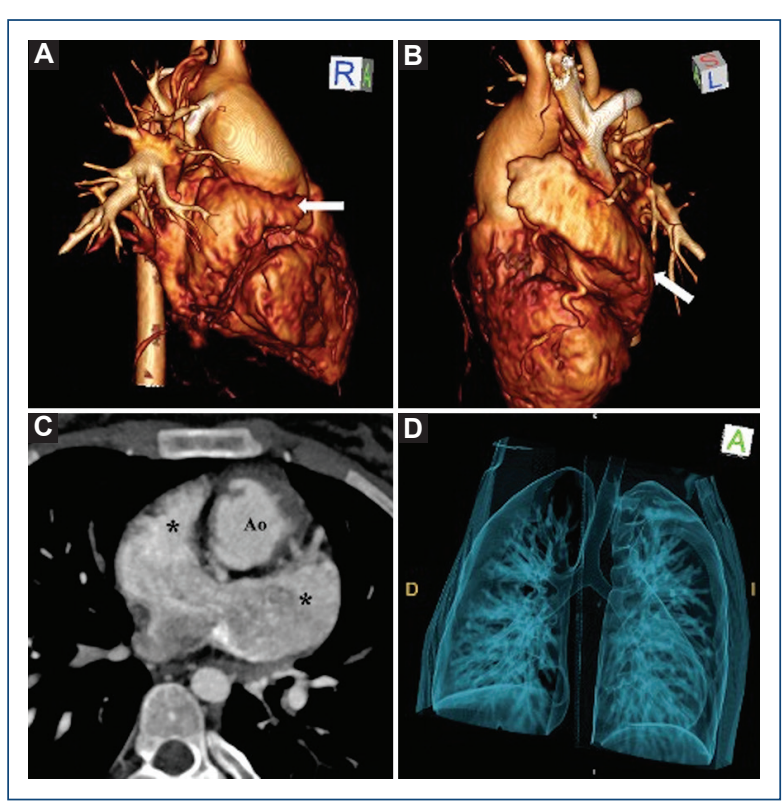

Figura 4. Reconstrucción $3 D$ que muestra (A, B) a nivel atrial la duplicación de una orejuela con características derechas (flecha) y ambos bronquios cortos y horizontales (D). Reconstrucción de proyección en máxima intensidad que muestra (C) ambos apéndices auriculares $\left({ }^{*}\right)$. Ao: aorta.

ventriculoarteriales suelen ser discordantes hasta en el $62 \% 21$. En cuanto al levomorfismo (Fig. 5), las estructuras bilaterales tienen características izquierdas, como bronquios morfológicamente izquierdos, ambos pulmones bilobulados y atrios izquierdos, por lo que no habrá crista terminalis ni nodo sinusal. Con la ausencia de un atrio morfológicamente derecho, la vena cava inferior no puede conectarse con el plano atrial y llega al corazón a través de la vena cava superior vía vena ácigos,
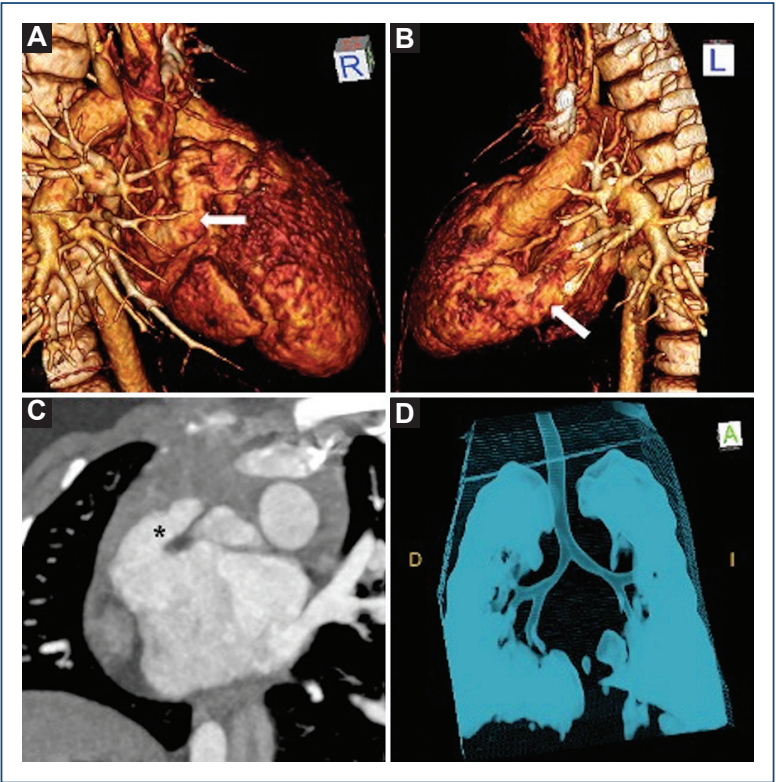

Figura 5. Reconstrucción 3D que muestra (A, B) apéndices auriculares de características izquierdas (flecha) y ambos bronquios largos (D). Reconstrucción de proyección en máxima intensidad que muestra (C) forma digitiforme típica de una orejuela morfológicamente izquierda(*) en posición topográfica derecha.

existiendo ausencia del segmento suprarrenal de la vena cava inferior y el segmento infrarrenal se continúa con la vena ácigos o hemiácigos ${ }^{26}$. Es importante destacar que, al existir esta interrupción del sistema venoso inferior, las venas suprahepáticas pueden también drenar directamente al atrio derecho en entre el $14 \mathrm{y}$ el $17 \%$ de los casos $^{21}$. Las venas pulmonares pueden estar conectadas de modo simétrico al atrio común, dos al atrio topográficamente a la derecha y dos al atrio 
topográficamente a la izquierda. Hay prevalencia de conexión auriculoventricular biventricular (65\%) con válvulas auriculoventriculares independientes en la mayoría de los casos (70\%); la conexión ventriculoarterial suele ser concordante $(45 \%)^{20}$. De forma extracardiaca está principalmente asociado con poliesplenia, caracterizada por la presencia de múltiples bazos pequeños que en masa se aproximan a la de un bazo normal ${ }^{25}$.

En último lugar el cateterismo cardiaco, al igual que los métodos anteriores, se puede utilizar como un complemento sobre todo para una caracterización de la anatomía vascular, además de dar un acercamiento a la respuesta fisiológica en relación con la vasculatura pulmonar en los casos de mayor edad con una cardiopatía estructural ${ }^{6}$.

Finalmente debemos tener en cuenta tres escenarios al momento del diagnóstico: primero, el predominio del dextromorfismo frente al levomorfismo $0^{9,27}$, en segundo lugar, a pesar de un abordaje diagnóstico con todos los instrumentos al alcance, la determinación precisa del diagnóstico en ocasiones resulta un desafío, incluso con la visualización directa de la morfología auricular durante el evento quirúrgico $0^{9} \mathrm{y}$, tercero, los pacientes con isomorfismo cardiaco se asocian generalmente a otras alteraciones extracardiacas, por lo que abordajes diagnósticos complementarios como el ultrasonido abdominal y estudios de imagen contrastados a nivel gastrointestinal, entre otros, se deben considerar para un apropiado abordaje en función de los hallazgos en la evaluación inicial.

\section{Manejo anestésico}

El manejo anestésico en estos pacientes representa un reto debido a la gran diversidad en la presentación clínica ${ }^{28}$. La valoración preanestésica de estos pacientes deberá incluir un minucioso análisis de las características morfológicas del paciente, para prever posibles complicaciones relacionadas con las múltiples malformaciones, por lo que mencionaremos puntos primordiales.

\section{Vía aérea}

Los pacientes con isomorfismo pueden llegar a cursar con alteraciones morfológicas de la línea media ( $45 \%$ en dextromorfismo y $52 \%$ en levomorfismo), no es extraño que se presenten con atresia de coanas, fístulas traqueoesofágicas, micrognatia, labio y paladar hendido ${ }^{29}$, debiendo realizarse una revisión de la cavidad oral y nasal previa al procedimiento anticipando el riesgo de una vía aérea difícil y de broncoaspiración durante la instrumentación de esta.

\section{Sistema digestivo}

Las malformaciones gastrointestinales, principalmente en la forma de malrotación intestinal, se presentan entre el 40 y el $90 \%$ de la población con isomorfismo ${ }^{30}$, condicionados a presentar un cuadro de obstrucción intestinal en el primer año de vida y condicionan un riesgo incrementado de presentar aspiración, alteraciones hidroelectrolíticas y alteraciones metabólicas en el ámbito perioperatorio ${ }^{28-31}$.

\section{Alteraciones pulmonares}

Las malformaciones en el retorno venoso pulmonar son la norma en los pacientes con dextromorfismo con la consiguiente hipertensión pulmonar secundaria; sin embargo, existen alteraciones pulmonares que van más allá de las alteraciones vasculares pulmonares. La discinesia ciliar primaria (DCP) consiste en una alteración intrínseca en la movilidad de las estructuras ciliares de diversos tejidos, especialmente en el epitelio respiratorio, alterando el aclaramiento de las secreciones del tracto respiratorio, con el consecuente desarrollo de dificultad respiratoria, sinusitis, bronquiectasias y neumonía secundaria a la pobre aclaración de secreciones mucociliares ${ }^{32}$. La estrecha relación que existe entre las alteraciones morfológicas en la lateralidad y la presencia de $\mathrm{DCP}$, así como la función indispensable que realiza la función ciliar en el nodo embrionario para la adecuada diferenciación en la lateralidad izquierda-derecha han llevado a la búsqueda de un origen genético que ligue ambas entidades ${ }^{33}$. Así, Nakhle, et al. (2012), encontraron que el $42 \%$ de los pacientes con cardiopatías congénitas asociadas a isomorfismo cuentan con algún grado de disfunción ciliar ${ }^{34}$. En una revisión retrospectiva por Kennedy, et al. (2007), el $76 \%$ de los casos presentaron dificultad respiratoria neonatal y el $100 \%$ de los pacientes mayores de 18 años tuvieron antecedente de bronquiectasias ${ }^{33}$. Dichas características de los patrones en la motilidad ciliar, aunado a las alteraciones en la vasculatura pulmonar, condicionan que la ventilación mecánica postoperatoria en estos pacientes se vea prolongada en hasta el $20 \%$ de los $\operatorname{casos}^{35}$.

\section{Alteraciones vasculares}

Existe una persistencia de vena cava superior izquierda en entre el 46 y el $71 \%$ de los casos ${ }^{21,36,37}$, así como una interrupción en la vena cava inferior en cerca del $80 \%$ de los pacientes con levomorfismo $6,8,21,29$, por 
lo que el acceso venoso femoral es preferido para la colocación de catéter venoso central, como lo es durante los procedimientos de derivación cavopulmonar superior o derivación cavopulmonar total, siendo importante considerar que el drenaje de dicho catéter venoso se hará en primera instancia hacia la vena ácigos y posteriormente drenará hacia el atrio a través de la vena cava superior ${ }^{38}$.

\section{Alteraciones del ritmo cardiaco}

Dependiendo la lateralidad, el paciente podrá presentar alteraciones en el ritmo cardiaco, que van desde un bloqueo auriculoventricular completo hasta taquicardia auricular, ventricular o de la unión $n^{1,6,7,39}$. Estas alteraciones se presentan debido a que en los isomorfismos, la topología auricular, así como los nodos y vías de conducción se ven severamente afectadas; presentando en el caso dextromorfismo la duplicación del nodo sinoauricular, mientras que en los casos de levomorfismo el nodo sinoauricular puede presentarse de manera hipoplásica o incluso estar ausente ${ }^{40,41}$. Durante el transoperatorio se debe contar en todo momento con las herramientas necesarias para cardioversión eléctrica, desfibrilación o estimulación de marcapasos.

El manejo intraoperatorio de cada paciente se deberá personalizar a manera de cubrir las necesidades fisiológicas y las variaciones anatómicas de cada caso, ya que el manejo de cada uno de los diferentes estadios para llegar a una fisiología univentricular, así como para una corrección biventricular, difieren sustancialmente entre ellos.

Los pacientes con isomorfismo cardiaco por sus características representan un manejo anestésico de alta complejidad, por lo tanto, se deberá recurrir al monitoreo invasivo de la presión arterial y de la presión venosa central para evaluar de manera directa las presiones de llenado auriculares ${ }^{28}$. Aquellos pacientes a quienes se llevará a un procedimiento de paliación univentricular deberán mantener una «circulación equilibrada», con un delicado equilibrio entre el flujo pulmonar, y por consiguiente el contenido arterial de oxígeno, y el flujo sistémico, que dependerá del robo de flujo sanguíneo a la vasculatura pulmonar secundario a los cambios en las resistencias vasculares pulmonares. Una vez realizada la paliación univentricular, el flujo pulmonar es de naturaleza pasiva, por lo que mantener un adecuado volumen intravascular y disminuir los condicionantes de un aumento en las resistencias vasculares pulmonares o en la presión intratorácica son herramientas indispensables para optimizar el flujo transpulmonar. El flujo pulmonar y la oxigenación arterial sistémica se ven severamente afectadas por la interrelación entre la presión en la arteria pulmonar, la presión venosa pulmonar y las resistencias vasculares pulmonares. La saturación arterial sistémica de oxígeno esperada es del $75-85 \%$.

\section{Tratamiento quirúrgico}

El tratamiento de los pacientes con diagnóstico de isomorfismo es variado y puede ir desde la cirugía paliativa en vista de una fisiología univentricular hasta una cirugía de corrección total para una reparación biventricular y esto será en función de la magnitud y gravedad de las anomalías cardiacas y de las lesiones extracardiacas que pueda tener asociadas ${ }^{1}$. Por lo anterior la mortalidad sigue siendo alta en este grupo de pacientes ${ }^{1}$ y aún más cuando existe asociación de factores de riesgo como edad neonatal, válvula auriculoventricular única e insuficiente, drenaje venoso pulmonar anómalo y alteración en las ramas de las arterias pulmonares ${ }^{39-42}$.

En caso de pacientes con fisiología univentricular, donde la mayoría tienen dextromorfismo, el tratamiento es disminuir la sobrecarga de volumen para el único ventrículo funcional, de esta manera la cirugía busca descongestionar esta carga de trabajo de manera progresiva y en algún momento, de ser posible, separar los dos circuitos, pulmonar y sistémico, llevando de una circulación paralela a una circulación en serie ${ }^{43,44}$. Los procedimientos paliativos son diversos y se plantean en función de la anatomía variable de esta patología, por lo que se puede llevar a cirugía para la creación de una fístula sistemicopulmonar en los casos de obstrucción en la circulación pulmonar o llevar a una cirugía de bandaje de la arteria pulmonar en los casos de sobrecirculación pulmonar ${ }^{1,43,44}$ y de manera preferente en la etapa neonatal. En un nuevo tiempo, la posibilidad de una derivación cavopulmonar bidireccional superior entre los 3 a 12 meses permite una mejor fuente de flujo sanguíneo pulmonar y con esto una mejor oxigenación, todo en consideración de las nuevas resistencias vasculares y la distensibilidad ventricular que el paciente posee ${ }^{1,44}$, para finalmente ser llevado a una derivación cavopulmonar total, la cual va a permitir una derivación del sistema venoso sistémico inferior hacia la circulación pulmonar, esto regularmente a partir de los 4 años de edad, para una mejor adaptación al nuevo sistema de circulación sanguínea. 
Por otro lado, la reparación biventricular puede ser posible en pacientes con levomorfismo, ya que las malformaciones cardiacas en la mayoría de los casos coexisten con dos ventrículos susceptibles de manejar los circuitos pulmonar y sistémico. En estos casos los defectos del tabique auriculoventricular, anomalías del retorno venoso sistémico y pulmonar con conexiones ventriculoarteriales concordantes son frecuentes, por lo que la reparación puede ser llevada a cabo, pero sin olvidar que puede existir durante su seguimiento cierto grado de insuficiencia auriculoventricular, lo que puede conllevar reoperaciones ${ }^{1} \mathrm{y}$, aunque la mortalidad en esta estrategia es menor a la univentricular, hasta el $21.4 \%$ de los pacientes con levomorfismo llevados a reparación biventricular pueden fallecer ${ }^{9}$.

\section{Financiamiento}

No se recibió ningún patrocinio para llevar a cabo este artículo.

\section{Conflicto de intereses}

Los autores declaran no tener ningún conflicto de intereses.

\section{Responsabilidades éticas}

Protección de personas y animales. Los autores declaran que para esta investigación no se han realizado experimentos en seres humanos ni en animales.

Confidencialidad de los datos. Los autores declaran que han seguido los protocolos de su centro de trabajo sobre la publicación de datos de pacientes.

Derecho a la privacidad y consentimiento informa-

do. Los autores han obtenido el consentimiento informado de los pacientes y/o sujetos referidos en el artículo. Este documento obra en poder del autor de correspondencia.

\section{Bibliografía}

1. Kim SJ. Heterotaxy syndrome. Korean Circ J. 2011;41(5):227-32.

2. Loomba RS, Hlavacek AM, Spicer DE, Anderson RH. Isomerism or heterotaxy: which term leads to better understanding? Cardiol Young. 2015;25(6):1037-43

3. Icardo JM, García Rincón JM, Ros MA. Congenital heart disease, heterotaxia and laterality. Rev Esp Cardiol. 2002;55(9):962-74

4. Calderón-Colmenero J, Cervantes-Salazar JL, Curi-Curi PJ, Ramírez-Marroquín S. Problemática de las cardiopatías congénitas en México. Propuesta de regionalización. Arch Cardiol Mex. 2010;80(2):133-40.

5. Cervantes-Salazar J, Calderón-Colmenero J, Ramírez-Marroquín J, Palacios-Macedo A, Bolio-Cerdán A, Vizcaíno-Alarcón A, et al. El Registro Mexicano de Cirugía Cardiaca Pediátrica. Primer informe. Evid Med Invest Salud. 2014;7(2):56-62.

6. Madrigal JS, Bonilla AC, Sánchez JE. Heterotaxia: Situs ambiguo, síndrome de Ivermark o síndrome de asplenia-poliesplenia. Rev Clin Esc Med. 2019;9(2):70-6.
7. Attie F. Diagnóstico anatómico de las cardiopatías congénitas. Arch Cardiol Mex. 2004;74(Suppl 1):13-7.

8. Carro Hevia A, Santamarta Liébana E, Martín Fernández M. Síndrome de heterotaxia. Cardiocore. 2011;46(2):e23-e26.

9. Alongi AM, Kirklin JK, Deng L, Padilla L, Pavnica J, Romp RL, et al. Surgical Management of Heterotaxy Syndrome: Current Challenges and Opportunities. World J Pediatr Congenit Heart Surg. 2020;11(2):166-76.

10. Banka P, Adar A, Schaetzle B, Sleeper LA, Emani S, Geva T. Changes in prognosis of heterotaxy syndrome over time. Pediatrics. 2020;146(2):e20193345.

11. Friedberg MK, Silverman NH, Moon-Grady AJ, Tong E, Nourse J, Sorenson $\mathrm{B}$, et al. Prenatal detection of congenital heart disease. J Pediatr. 2009;155(1):26-31.e1.

12. Pepes $\mathrm{S}$, Zidere $\mathrm{V}$, Allan LD. Prenatal diagnosis of left atrial isomerism. Heart. 2009:95(24):1974-7.

13. Lai WW, Geva T, Shirali GS, Frommelt PC, Humes RA, Brook MM, et al.; Task Force of the Pediatric Council of the American Society of Echocardiography; Pediatric Council of the American Society of Echocardiography. Guidelines and standards for performance of a pediatric echocardiogram: a report from the Task Force of the Pediatric Council of the American Society of Echocardiography. J Am Soc Echocardiogr. 2006;19(12):1413-0

14. Acherman RJ, Evans WN. Persistent right umbilical vein in isomerism. Prenat Diagn. 2019;39(13):1220-4.

15. Geva T, Vick GW 3rd, Wendt RE, Rokey R. Role of spin echo and cine magnetic resonance imaging in presurgical planning of heterotaxy syndrome. Comparison with echocardiography and catheterization. Circulation. 1994:90(1):348-56.

16. Han BK, Rigsby CK, Hlavacek A, Leipsic J, Nicol ED, Siegel MJ, et al. Computed tomography imaging in patients with congenital heart disease Part I: Rationale and utility. An expert consensus document of the Society of Cardiovascular Computed Tomography (SCCT): Endorsed by the Society of Pediatric Radiology (SPR) and the North American Society of Cardiac Imaging (NASCl). J Cardiovasc Comput Tomogr. 2015;9(6):475-92.

17. van Praagh R, David I, Wright GB, van Praagh S. Large RV plus small LV is not single RV. Circulation. 1980;61:1057-9.

18. Ghosh S, Yarmish G, Godelman A, Haramati LB, Spindola-Franco H. Anomalies of visceroatrial situs. Am J Roentgenol. 2009;193(4):1107-17.

19. Wolla CD, Hlavacek AM, Schoepf UJ, Bucher AM, Chowdhury S. Cardiovascular manifestations of heterotaxy and related situs abnormalities assessed with CT angiography. J Cardiovasc Comput Tomogr. 2013; $7(6): 408-16$.

20. Frescura C, Ho SY, Giordano M, Thiene G. Isomerism of the atrial appendages: morphology and terminology. Cardiovasc Pathol. 2020; 47:107205.

21. Uemura H, Ho SY, Devine WA, Kilpatrick LL, Anderson RH. Atrial appendages and venoatrial connections in hearts from patients with visceral heterotaxy. Ann Thorac Surg. 1995;60(3):561-9.

22. Bierhals AJ, Rossini S, Woodard PK Javidan-Nejad C, Billadello Ju, Bhalla $S$, et al. Segmental analysis of congenital heart disease: putting the "puzzle" together with computed tomography. Int J Cardiovasc Imaging. 2014;30(6):1161-72.

23. Cano-Zárate R, Meave-González A. Análisis por tomografía y resonancia magnética de las cardiopatías congénitas a través del sistema secuencial segmentario. An Radiol Mex. 2014;13(2):117-27.

24. Balan A, Lazoura O, Padley SP, Rubens M, Nicol ED. Atrial isomerism: a pictorial review. J Cardiovasc Comput Tomogr. 2012;6(2):127-36.

25. Perloff JK. The cardiac malpositions. Am J Cardiol. 2011;108(9):1352-61.

26. Thiene G, Frescura C. Asplenia and polysplenia syndromes: time of successful treatment and updated terminology. Int J Cardiol. 2019;274:117-9.

27. Loomba RS, Nijhawan K, Anderson R. Impact of era, type of isomerism, and ventricular morphology on survival in heterotaxy: Implications for therapeutic management. World J Pediatr Congenit Heart Surg. 2016;7(1):54-62.

28. Williams GD, Feng A. Heterotaxy syndrome: implications for anesthesia management. J Cardiothorac Vasc Anesth. 2010;24(5):834-44.

29. Ticho BS, Goldstein AM, van Praagh R. Extracardiac anomalies in the heterotaxy syndromes with focus on anomalies of midline-associated structures. Am J Cardiol. 2000;85(6):729-34.

30. Hill SJ, Heiss KF, Mittal R, Clabby ML, Durham MM, Ricketts R, et al. Heterotaxy syndrome and malrotation: does isomerism influence risk and decision to treat. J Pediatr Surg. 2014;49(6):934-7; discussion 937.

31. Lim JS, McCrindle BW, Smallhorn JF, Golding F, Caldarone CA, Taketazu $\mathrm{M}$, et al. Clinical features, management, and outcome of children with fetal and postnatal diagnoses of isomerism syndromes. Circulation. 2005;112(16):2454-61.

32. Knowles MR, Zariwala M, Leigh M. Primary ciliary dyskinesia. Clin Chest Med. 2016:37(3):449-61.

33. Kennedy MP, Omran H, Leigh MW, Dell S, Morgan L, Molina PL, et al. Congenital heart disease and other heterotaxic defects in a large cohort of patients with primary ciliary dyskinesia. Circulation. 2007:115(22):2814-21.

34. Nakhleh N, Francis R, Giese RA, Tian X, Li Y, Zariwala MA, et al. High prevalence of respiratory ciliary dysfunction in congenital heart disease patients with heterotaxy. Circulation. 2012;125(18):2232-42. 
35. Swisher M, Jonas R, Tian X, Lee ES, Lo CW, Leatherbury L. Increased postoperative and respiratory complications in patients with congenital heart disease associated with heterotaxy. J Thorac Cardiovasc Surg. 2011:141(3):637-44.e1-3.

36. Phoon CK, Neill CA. Asplenia syndrome: insight into embryology through an analysis of cardiac and extracardiac anomalies. Am J Cardiol. 1994;73(8):581-7.

37. Peoples WM, Moller JH, Edwards JE. Polysplenia: a review of 146 cases. Pediatr Cardiol. 1983;4(2):129-37.

38. Sharma S, Devine W, Anderson RH, Zuberbuhler JR. Identification and analysis of left atrial isomerism. Am J Cardiol. 1987;60(14):1157-60.

39. Chen W, Ma L, Cui H, Yang S, Xia Y, Zou M, et al. Early- and middle-term surgical outcomes in patients with heterotaxy syndrome. Cardiology. 2016;133(3):141-6.
40. McGovern E, Kelleher E, Potts JE, O'Brien J, Walsh K, Nolke L, et al. Predictors of poor outcome among children with heterotaxy syndrome: a retrospective review. Open Heart. 2016;3(2):e000328.

41. Yan S, Jianpeng W, Xin Q, Minghui Z, Li Z, Hao W. Right atrial isomerism in children older than 3 years. Springerplus. 2016;5(1):1372.

42. Baban A, Cantarutti N, Adorisio R, Lombardi R, Calcagni G, Piano Mortari $\mathrm{E}$, et al. Long-term survival and phenotypic spectrum in heterotaxy syndrome: A 25-year follow-up experience. Int J Cardiol. 2018;268:100-5.

43. Hosseinpour AR, González-Calle A, Adsuar-Gómez A. ¿Qué queremos decir con el término univentricular? Cir Cardiov. 2015;22(4):193-4.

44. Serrano F, Caffarena JM. Cirugía del corazón univentricular en segundo estadio: técnicas y resultados. Cir Cardiov. 2008;15(4):351-60. 